\title{
Blinking suppression and intensity recurrences in single CdSe-oligo(phenylene vinylene) nanostructures: experiment and kinetic model
}

\author{
K T Early ${ }^{1}$, K D McCarthy ${ }^{1}$, N I Hammer ${ }^{1}$, M Y Odoi ${ }^{1}$, R Tangirala ${ }^{2}$, T Emrick $^{2}$ \\ and M D Barnes ${ }^{1 *}$ \\ ${ }^{1}$ George H. Richason, Jr. Chemistry Research Laboratory, Department of Chemistry, \\ University of Massachusetts, Amherst, Massachusetts 01003 \\ ${ }^{2}$ Department of Polymer Science and Engineering, University of Massachusetts, Amherst, \\ Massachusetts 01003 \\ E-mail: mdbarnes@chem.umass.edu
}

\begin{abstract}
We report time-resolved single molecule fluorescence imaging of individual CdSe quantum dots that are functionalized with oligomeric conjugated organic ligands. The fluorescence intensity trajectories from these composite nanostructures display both a strong degree of blinking suppression and interesting intensity fluctuations with recurrence times on the order of 10-60 seconds (quantified by Fourier transform analysis). In addition, Time-Tagged-TimeResolved (TTTR) fluorescence measurements of the fluorescence decay rate of individual hybrid nanostructures indicate significantly modified non-radiative decay rates relative to conventional ZnS-capped CdSe quantum dots. Modeling of fluorescence intensity trajectories using a diffusive reaction coordinate model shows that slow fluctuations in electron energies ( $1 \mathrm{Se}, 2 \mathrm{Pe})$ can give rise to the recurrences via modified Auger-assisted hole-trapping rates. We briefly discuss a possible ligand-induced mechanism for such behavior.
\end{abstract}

\footnotetext{
* Author to whom correspondence should be addressed
} 


\section{Introduction}

Semiconductor quantum dots (QDs) are well known for their size-tunable optical and electronic properties, broad optical absorption spectra, and narrow emission spectra that make them attractive in applications such as biological labeling [1], photovoltaic devices [2], and single photon emitters [3]. However, the issue of fluorescence intermittency (blinking) is problematic in many of these applications, and has been the subject of many recent experimental and theoretical studies[4-10]. A number of interesting features of QD photoluminescence have been well documented including inverse power law blinking kinetics [10, 11], statistical aging in intensity autocorrelation measurements $[9,12]$, radiative rate fluctuations [13], and spectral diffusion [14, 15]. Recently, we and others reported suppression of QD blinking behaviour, either as a result of coordination of the QD surface with suitable organic ligands $[13,16,17]$ or other environmental parameters $[18,19]$. The qualitative picture proposed for blinking suppression in these systems involves carrier trapping at the QD surface from electron-donating moieties, which in turn enhances the relative probability of radiative recombination within the QD.

We recently reported on the photoluminescence dynamics of CdSe quantum dots functionalized with para-phenylene vinylene oligomers (CdSe-OPV) [20]. These composite nanostructures (schematic illustration included as figure 1) exhibited enhanced spectral stability and significantly reduced blinking, with average dark-state dwell times on the order of $500 \mathrm{~ms}$, compared to tens of seconds for ZnS-capped quantum dots [21]. Time-resolved spectral studies of individual CdSe-OPV nanostructures showed that virtually all of the

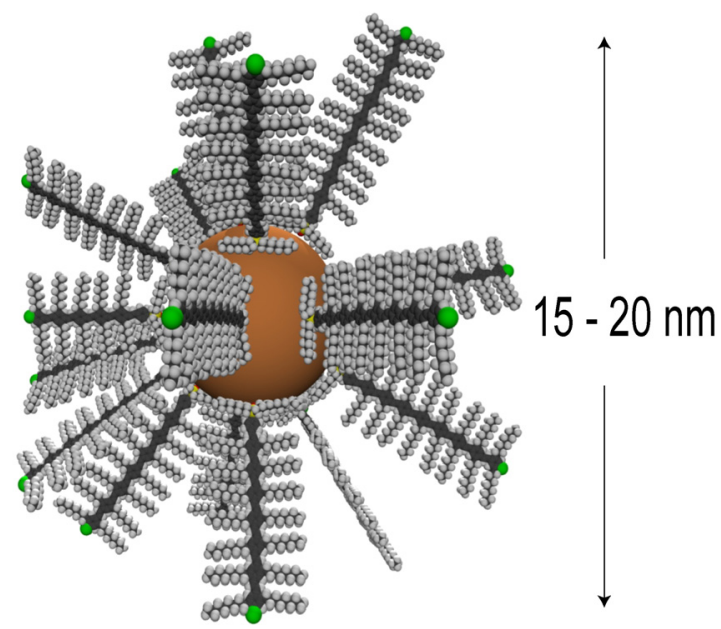

Figure 1. Schematic illustration of a CdSe-OPV composite nanostructure, as determined from sizecorrelated fluorescence measurements [20]. The QD diameter is 4.5 with $\sim 10 \%$ monodispersity. Composite particles range in size from $10-25 \mathrm{~nm}$, depending on ligand coverage 
photoluminescence is associated with the QD, indicating that excitations within the organic ligands are efficiently quenched by energy transfer and/or charge-transfer processes facilitated by the molecular architecture [17]. Here, we discuss the experimental observation and kinetic modeling of QD "flickering," where the fluorescence intensity trajectories show a broad distribution of bright states, and reveal the existence of recurrence features apparent in the fluorescence intensity trajectories of individual CdSe-OPV composite nanostructures. Finally, we present results of numerical simulation of photoluminescence intensity trajectories based on an extension of the diffusive coordinate (DC) model proposed recently by Frantsuzov and Marcus [22]. We show that such a model will yield a pseudo-periodic behaviour in the fluorescence intensity as a result of fluctuations of the coordinate $\varepsilon$ (the energy separation between $\mathrm{S}$ and $\mathrm{P}$ excitonic states).

\section{Experimental}

CdSe-OPV nanostructures were prepared by polymerization of aromatic vinyl bromides from the surface of functionalized quantum dots [20], resulting in a distribution of nanostructure sizes [17]. All dilutions were made in high purity tetrahydrofuran (THF) to single molecule concentration $(\sim 100$

$\mathrm{pM})$ and deposited on plasma-cleaned glass coverslips $\left(<1\right.$ quantum $\left.\operatorname{dot} / \mu \mathrm{m}^{2}\right)$. Fluorescence imaging was performed under ambient conditions on an inverted microscope (Nikon TE300) in total internal reflection geometry through a 1.4 NA oil objective. Emitted light was collected through the same objective, filtered using a dichroic beamsplitter and filter set and sent to an EMCCD array (Princeton Instruments/Acton PhotonMax). Height signatures for individual CdSe-OPV nanostructures were obtained by AFM in TappingMode (Digital Instruments Bioscope) and correlated with fluorescence images as described previously [17].

Time-Tagged-Time-Resolved (TTTR) measurements were performed using a $440 \mathrm{~nm}$ Picoquant pulsed diode picosecond laser operating at $10 \mathrm{MHz}$ and with a TimeHarp-200 time-todigital converter operating at 34-ps resolution. An avalanche photodiode, APD, (PerkinElmer SPCM- 
$\mathrm{AQR}-14, \approx 50 \mathrm{cps}$ dark count rate) was used as a single-photon detector whose output was read. A precision servo-controlled stage (ASI MS-2000) was employed to position the sample in order to image fluorescence from a single particle with coordinates registered with the APD. Thus, every particle is probed with approximately the same excitation intensity. The overall photon detection efficiency at the APD was estimated to be about $0.5 \%$; typical peak count rates (for CdSe/ZnS) were $\approx$ $4 \mathrm{kHz}$, corresponding to a nominal excitation efficiency/per pulse of $\approx 0.1$.

\section{Results}

\subsection{Single Nanostructure Experimental Results}

Figure 2 shows intensity traces and corresponding power spectra for three representative nanostructures of diameter (a,d) $16.0 \mathrm{~nm},(\mathrm{~b}, \mathrm{e}) 21.9 \mathrm{~nm}$, and (c,f) $23.9 \mathrm{~nm}$. As discussed previously, the CdSe-OPV nanoparticle size distribution derives from various degrees of surface coverage. As illustrated, the fluorescence trajectories of these particles exhibit well-defined recurrence times on scales of 10 - 50 seconds, which are reflected in the power spectrum for each particle. No such recurrences were observed from control trials using $20 \mathrm{~nm}$ dyedoped polystyrene nanospheres (Invitrogen, Molecular Probes Fluospheres) indicating that the recurrences are not associated with camera artefacts or laser intensity fluctuations.
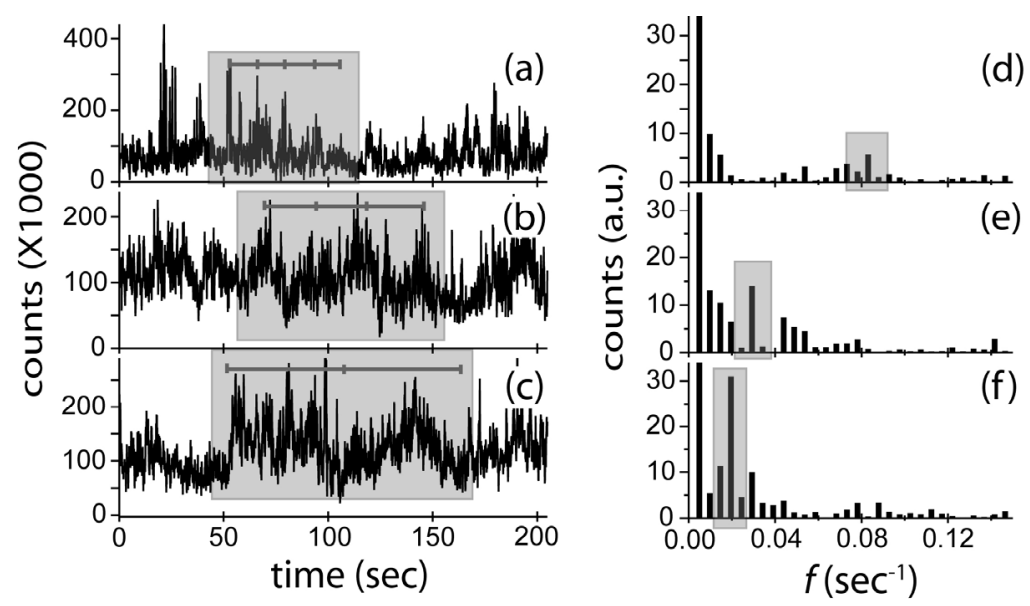

Figure 2. Representative photoluminescence Intensity trajectories and corresponding power spectra for three $\mathrm{CdSe}-\mathrm{OPV}$ nanostructures of diameter (a,b) 16.0, (c,d) 21.9, and (e,f) $23.9 \mathrm{~nm}$. Recurrence features are highlighted in intensity trajectories along with corresponding spectral components.

Figure 2 also shows the growth in low frequency spectral components with increasing nanostructure diameter that were present in some degree for every nanostructure imaged. Evidence of 
the characteristic frequency scaling with ligand coverage will be discussed elsewhere. For sparsely functionalized nanostructures (evidenced by surface height signatures comparable to bare QDs), such as that represented in figure $2 \mathrm{~d}$, the fluorescence flickering is characterized largely by low amplitude, high frequency noise. As surface coverage increases (figure 2f), large amplitude, low frequency peaks (longer recurrence times) become evident in the power spectrum. This trend indicates an oscillating relaxation rate which is highly sensitive to the presence of OPV ligands coordinated to the QD surface.

Figure 3 shows similar fluorescence intensity trajectories obtained from individual CdSe/ZnS QDs and CdSe-OPV nanostructures in TTTR mode. Briefly, TTTR is a time-correlated single-photon counting technique whereby each fluorescence photon is identified by two time stamps: the relative time with respect to last excitation pulse, and the time relative to the start of data acquisition (50 ns clock resolution). Thus, TTTR allows access to fluorescence decay rate information within any arbitrary segment of a fluorescence intensity trajectory and provides a way correlating fluorescence intensity with measured decay rate. Such techniques have been recently used to characterize intensity fluctuations in $\mathrm{CdSe} / \mathrm{ZnS}$ QDs in terms of an approximate continuum of bright-levels [13, 23]. Our TTTR data for individual CdSe/ZnS QDs show the same positive correlation between fluorescence intensity and fluorescence lifetime as observed by Yang and co-
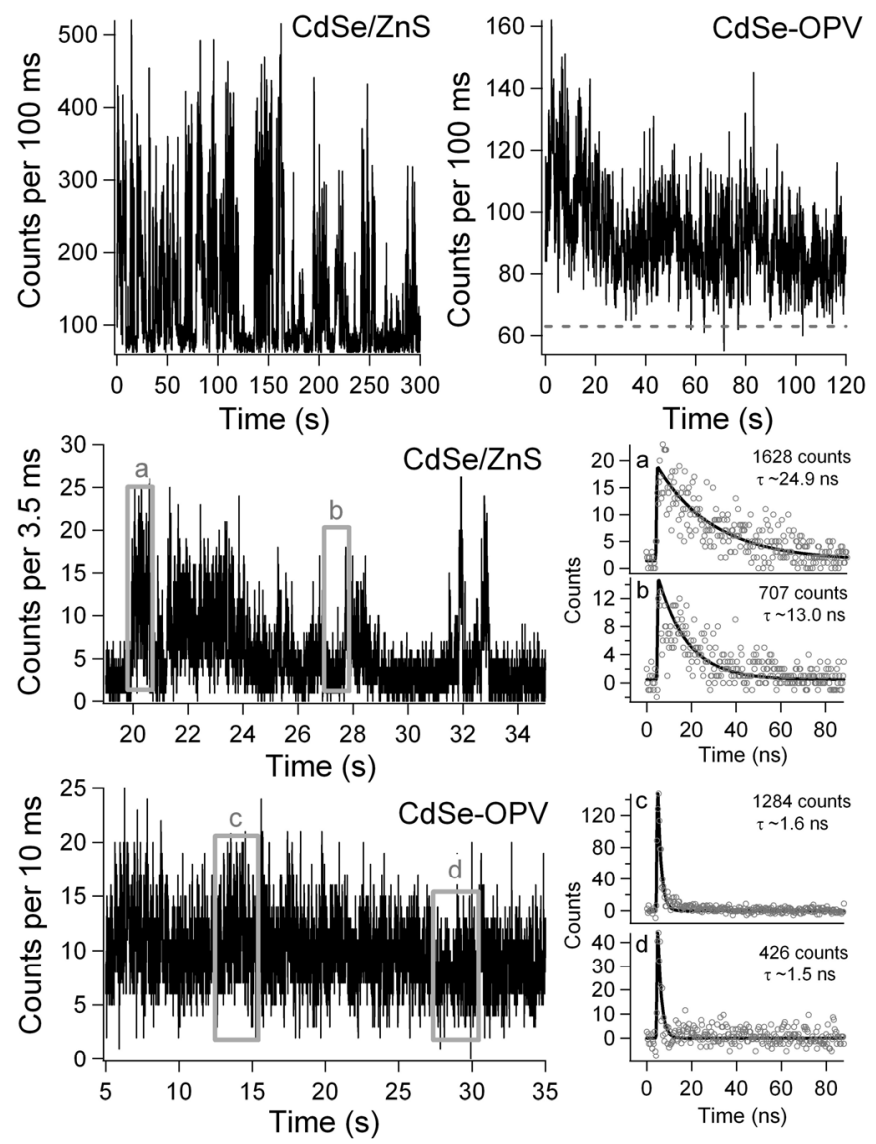

Figure 3. TTTR intensity trajectories and radiative lifetime measurements of CdSe/ZnS and CdSe-OPV QDs. The lifetimes for the indicated time segments were fit to single exponential decay functions. 
workers [23]. That is, the peak fluorescence intensities are associated with a relatively long fluorescence lifetime $(\approx 20 \mathrm{~ns})$ - presumably a good approximation of the pure radiative lifetime of the dot and lower fluorescence intensities are associated with shorter fluorescence lifetimes indicating a competing non-radiative decay mechanism. This feature of QD luminescence has previously been observed and discussed by others.

For ZnS-capped QDs, the measured lifetime during a period of highest-intensity luminescence was determined here to be about $25 \mathrm{~ns}$, in agreement with the earlier observations by both Mews and Yang $[23,24]$. Intermediate intensities $(\approx 1-2 \mathrm{kHz}$ count rates) were associated with smaller lifetimes $(7-12 \mathrm{~ns})$. This effect can be understood in terms of fluctuations in the non-radiative decay rate $\left(k_{N R}\right)$. When $k_{N R}$ is small compared to the radiative decay rate $k_{R}$, efficient cycling between ground and excited states associated with photon emission takes place, resulting in high fluorescence intensity. In regions where $k_{N R} \geq k_{R A D}$, the number of photons emitted decreases due to competition from the nonradiative pathway, but the measured decay rate $\left(k_{D}=k_{N R}+k_{R}\right)$ increases thus decreasing the measured fluorescence lifetime and decreasing the fluorescence intensity.

Intensity trajectories from CdSe-OPV nanostructures probed here by TTTR show similar intensity oscillations and blinking suppression as probed by CW illumination represented in figure 2 . The same trend observed for the ZnS-capped QDs was observed for individual CdSe-OPV nanostructures, however, with much shorter measured lifetimes (obtained during periods of both high and low intensity emission) than that of the CdSe/ZnS. Typical lifetimes within $500 \mathrm{~ms}-5$ second time windows were between 1.6 and $2.8 \mathrm{~ns}$. Assuming a value of $25 \mathrm{~ns}$ for the pure radiative lifetime of the QD exciton, our measurements suggest that non-radiative decay dominates with values of $k_{N R} \leq$ $0.3 \mathrm{~ns}^{-1}$. In the following section, we present numerical simulations based on the DC model that provide new physical insight into the mechanism of blinking suppression in QD systems, and suggest a mechanism for intensity recurrences as well. 


\subsection{The Diffusive Reaction Coordinate Model of Quantum Dot Photoluminescence Fluctuations}

Many models have been proposed to explain blinking rates in quantum dot systems, ranging from photoinduced charging models [4] to multiply charged surface site models [25]. However, the DC model proposed by Frantsuzov and Marcus [22] is attractive for describing CdSe-OPV emission behaviour due to its incorporation of a diffusive non-radiative coordinate in a harmonic potential. Coupling of this diffusive motion to non-radiative decay rates will naturally lead to bounded, slowly evolving trajectories in the photoluminescence intensity, as observed in our experimental data. Here, we show that an extension of this model, with some changes assumed in the form for non-radiative relaxation due to Auger-assisted hole trapping, yields the observed trend in recurrence time scale through variation of two parameters that may be related to the presence of complex ligands such as

OPV. In the DC model, light-induced diffusional fluctuations in the quantum dot "environment" (that is, all degrees of freedom aside from the two-level system describing ground and excited states) have a strong influence on hole-trapping processes that act as non-radiative relaxation processes.

In this picture, the parameter $\varepsilon=E_{1 P e}-E_{1 S e}$ (the difference in energies between the lowest two above-gap electronic states) takes on the role of a slowly diffusing reaction coordinate that describes (by proxy) structural or electronic changes at the surface of the quantum dot. Such changes are assumed to be dominated by light-induced conformational changes occurring at the surface during absorption and radiative relaxation processes. By way of conservation of energy, $\varepsilon$ (in conjunction with the lineshape of the surface states) dictates directly the rate of Auger-assisted trapping of the excitonic hole in an intra-gap band of surface states, leading to efficient non-radiative relaxation of the exciton and subsequent modulation of the photoluminescence intensity.

Figure 4a illustrates the processes that dictate photoluminescence intensity in the DC model, as well as the role of $\varepsilon$ in modulating the hole trapping process, as originally described by Frantsuzov and Marcus [22]. Auger-assisted hole trapping is assumed to split the dynamics into a non-radiative path (light blue arrows) that competes kinetically with the radiative path (red arrows). It is via the $\varepsilon$ - 
dependence of the Auger hole-trapping rate that diffusive dynamics of the environment (surface, etc.)

influence the importance of the non-radiative path. In figure $4 \mathrm{~b}$, we have plotted the parabolic diffusion potential alongside the trapping rates for the two cases. We find that a Breit-Wigner or similarly slowly varying form of the hole-trap lineshape function is essential for recovering the experimentally observed intensity fluctuations. The grey-scale background is designed to indicate relative luminescence brightness correlated with the random variable $\left(\varepsilon-\varepsilon_{0}\right)$. From this picture one can see how the sharp cut-off of the Gaussian form leads to high-contrast "on-off” behaviour, while the more slowly varying Breit-Wigner form leads to a "flickering" behaviour, represented as a continuum of grey levels.

(a)

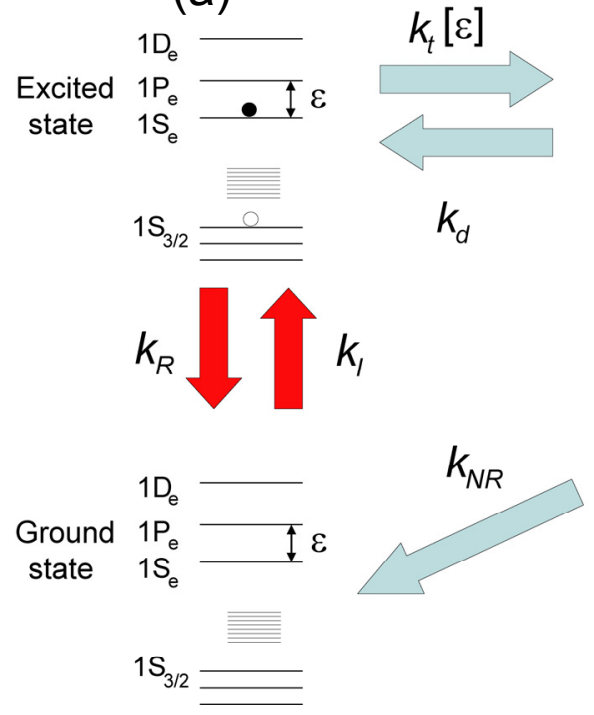

(b)

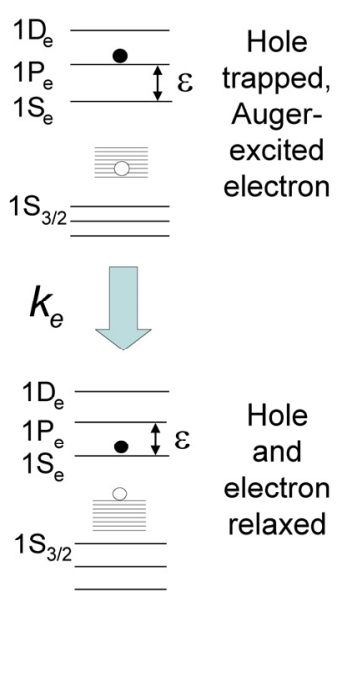

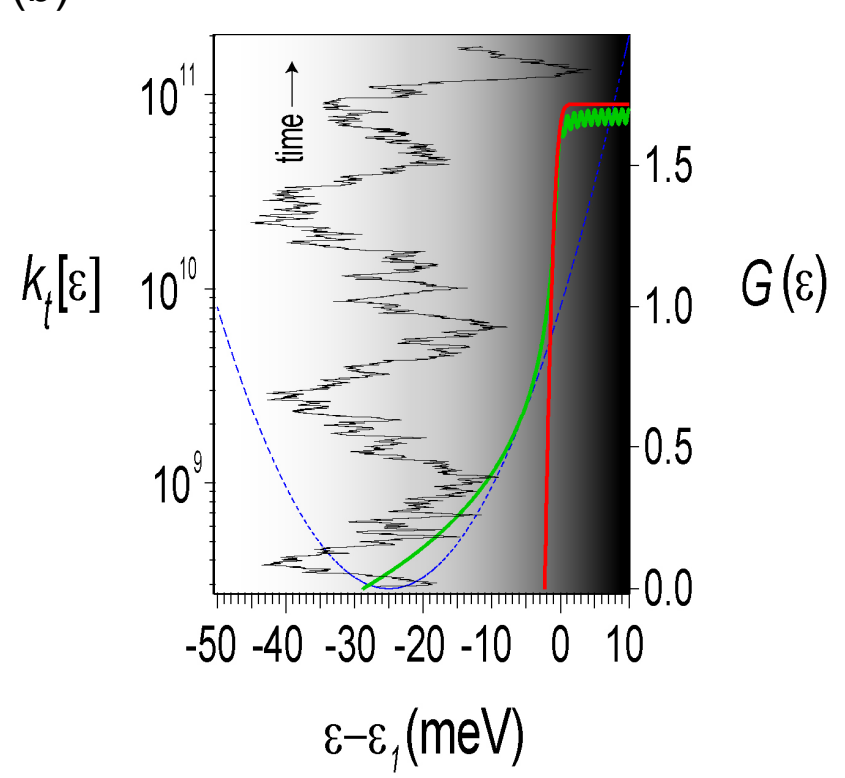

Figure 4 (a) QD states and kinetics relevant to the photoluminescence of CdSe-based nanostructures, following the DC model of Frantsuzov and Marcus. The photon absorption rate $k_{I}$ and radiative relaxation rate $k_{R}$ compete with the non-radiative path (light blue arrows) resulting in strong fluctuations in photoluminescence intensity. The rate for hole de-trapping is assumed to be similar to $k_{t}[\varepsilon] . k_{e}$ is the rate for phonon-assisted $1 P_{\mathrm{e}}$ to $1 S_{\mathrm{e}}$ transition. $k_{N R}$ is the rate for final non-radiative recombination of the electron-hole pair. (b) The parabolic potential (dotted blue trace, right axis) controlling diffusion of $\varepsilon$, superimposed on the Gaussian or strong-coupling (solid red trace, left axis) form and the Lorentzian or Breit-Wigner (solid green trace, left axis) form of the Auger-assisted hole-trapping rate. Regions of $\varepsilon$ corresponding to large values of $k_{t}\lceil\varepsilon]$ are associated with dark states. A simulation of bias walk in $\varepsilon$ is shown.

Tang and Marcus have used similar arguments to explain the ubiquitous phenomena of spectral diffusion in semiconductor quantum dots with some encouraging agreement with experiment 
[26]. In this case, it is the excitation energy $E_{e x}$ itself that undergoes diffusion, resulting in a drift of the emitted photon energy on time scales of seconds. The quasi-binary behaviour fluorescence intermittency via diffusion of $\varepsilon$, was shown to be closely associated with the functional form of the Auger-assisted hole-trapping rate on $\varepsilon$ that Frantsuzov and Marcus defined as [22]

$$
k_{t}[\varepsilon]=\alpha \sum_{i} \exp \left[-\frac{\left(\varepsilon-\varepsilon_{i}\right)^{2}}{\beta^{2}}\right]
$$

where the index $i$ runs over the $\sim 200$ trap states postulated to be formed by Se dangling bonds on the surface of the dot. Based on some reasonable parameters, the conclusion was that the diffusion of $\varepsilon$ occurs over large amplitudes that brings $\varepsilon$ into and out of the region of very large trapping rates. Visitations into these regions associated with large values of $k_{t}[\varepsilon]$ result in long dark periods, while excursions out of the region of large trapping rate yield the bright regions within the trajectory.

In order to explain the blinking suppression and the presence of long-time recurrence features in photoluminescence intensity, we must have either diffusion of $\varepsilon$ limited to amplitudes over which the Auger-assisted trapping rate is always in competition with the rate for radiative recombination, or a different, long-tailed form for the hole-trapping rates than assumed in [22]. We take the latter approach and find that the assumption of Breit-Wigner (Lorentzian) lineshapes and trapping rate

$$
k_{t}^{B W}[\varepsilon]=\alpha \sum_{i} \frac{\left(\frac{\beta}{2}\right)^{2}}{\left(\frac{\beta}{2}\right)^{2}+\left(\varepsilon-\varepsilon_{i}\right)^{2}}
$$

as opposed to the strong-coupling or Gaussian form assumed in (1), gives the necessary photoluminescence dynamics needed for extending the ideas of DC theory to CdSe-OPV nanostructures. We also assume a reduction in $\varepsilon_{0}$, the bare value of the energy difference $\mathrm{E}_{1 \mathrm{Pe}}-\mathrm{E}_{1 \mathrm{Se}}$. These "ansatz" alterations of the DC model for CdSe-OPV are entirely reasonable in light of the profound effect of the precise nature of the dative phosphine oxide bond to $\mathrm{Cd}$ on the dot electronic structure [27]. In general, OPV ligands present a complex electronic and photonic environment of the 
surface states; such an environment might alter the many-body transition matrix elements in such a way as to yield the necessarily broad form of the hole trapping rate needed for diffusive processes to leave their fingerprint [28].

The central idea behind our numerical simulation of fluorescence intensity trajectories is that the number of detected photons within a specific time window is inversely proportional to the overall decay rate expressed as a sum of radiative and non-radiative rates, $k_{R}$ and $k_{N R}$. The nonradiative rate constant that competes with $k_{R}$ is directly related to the instantaneous value of $\varepsilon$, so propagation of a diffusional trajectory in the coordinate $\varepsilon$ yields a model of the intensity trajectory. At each step, the steady state solution of the kinetic model of photoluminescence [22] is utilized to calculate the steady state value of the photoluminescence, given $\varepsilon$ as the input to the Auger-assisted trapping rate. All data presented assumes constant rates for photo-absorption (absorbed flux of $5.0 \times 10^{6}$ ), radiative lifetime (20 ns), rate for non-radiative recombination of trapped hole and electron $\left(\sim 10^{6} \mathrm{~s}^{-1}\right)$, and a fast, overall rate of the (hole-trapping/electron $1 P_{e}$ to $\left.1 S_{\mathrm{e}}\right)$ process $\left(3 \times 10^{11} \mathrm{~s}^{-1}\right)$. Also constant are the parameters characterizing Breit-Wigner trap state lineshapes $\left(\alpha=5.0 \times 10^{10} \mathrm{~s}^{-1}, \beta=1.0 \mathrm{meV}\right)$.

Figure 5 shows simulation results from parameters that yield reasonable agreement with our experimental intensity trajectory data. While the parameter space available to explore in the simulation is very large, we have strived to make the parameters agree as well as possible with estimates in [22]. The most notable deviation from the parameters in the original theory is a decreased diffusion amplitude $\Delta_{\varepsilon}$ (10-30 meV compared to $\sim 90 \mathrm{meV}$ in [22]). However, we stress that the most important factor
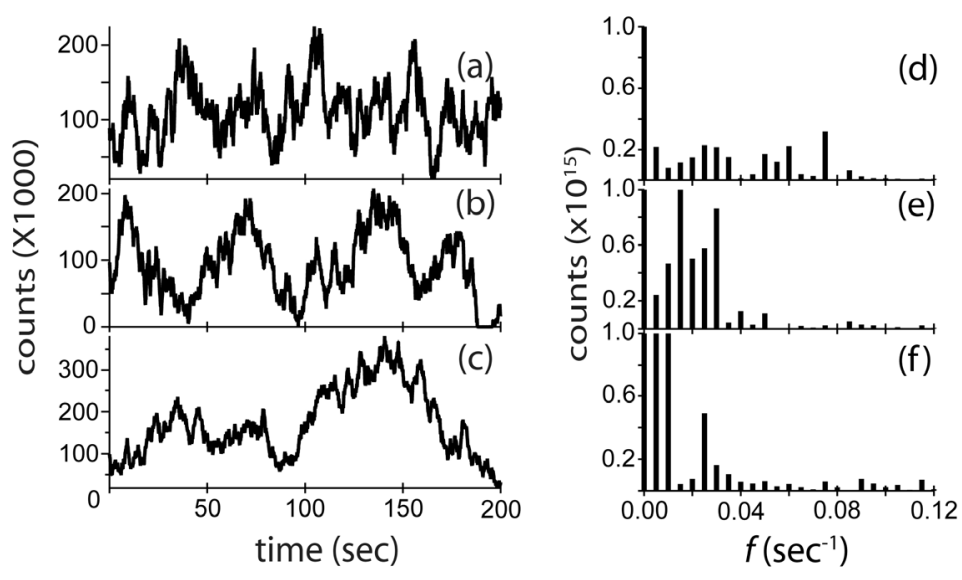

Figure 5. Simulation results for Frantsuzov-Marcus theory using Breit-Wigner form for Auger-assisted trapping rate. From top to bottom: $\Delta_{d} / a_{\varepsilon}=10 \mathrm{meV} / 2.0 \mu \mathrm{eV}, 25 \mathrm{meV} / 1.6 \mu \mathrm{eV}, 30 \mathrm{meV} / 1.5$ $\mu \mathrm{eV}$. Shown also are corresponding power spectra highlighting the specific recurrence features. 
for agreement with our experimental results is the assumed Breit-Wigner form for the Auger-assisted hole-trapping rate. Figure 5 shows that a simultaneous increase of $\Delta_{\varepsilon}$ and lowering of $a_{\varepsilon}$ (the size of the light-induced diffusive steps in $\varepsilon$ ), is a potential mechanism for the increased prominence of low frequency features in the photoluminescence time traces. Since this trend is associated with increased ligand coverage in our correlated AFM/fluorescence data, we speculate that the effects of the ligand environment on dot energetics may be involved in such a picture [29].

\section{Conclusions}

We find that observed periodic recurrences in the photoluminescence emission intensity from CdSe-OPV nanostructures, and blinking suppression, can be explained using a diffusive coordinate (DC) model similar to Frantsuzov and Marcus with a modified hole-trap lineshape function. We demonstrate via numerical simulation that a modified diffusive coordinate model can reproduce the periodicity and associated amplitude given a large dynamic range (over values of $\varepsilon$ ) for Auger-assisted hole-trapping. The result of this modification is a more slowly varying $k_{N R}$ which reduces - but does not extinguish - the fluorescence intensity. It is apparent that the important contributions to correctly simulate the photoluminescence dynamics in our CdSe-OPV data are the broader resonances of surface-hole trap states, resulting in a wide region of slowly varying Auger-assisted hole trapping, and constrained excursions of $\varepsilon$ to values where blinking to the "off" state can not be observed. The second of these contributions has two possible origins: a reduction in $\varepsilon_{0}$, the nominal value of the $1 P_{\mathrm{e}}-$ $1 S_{\mathrm{e}}$ energy difference, or the constrained diffusion of $\varepsilon$ to smaller values, or some combination of these two effects. At this point, it is difficult to pinpoint the precise role of the conjugated organic ligands in the modification of the hole-trap lineshape, but the mere presence of the observed recurrences strongly suggests a dynamically varying non-radiative transition rate. 


\section{Acknowledgments}

We would like to acknowledge support from the NSF-Sponsored Center for Heirarchical Manufacturing at UMass-Amherst, MRSEC, the US-DOE Basic Energy Sciences (05ER15695) and the Intelligence Community Postdoctoral Fellowship Program.

\section{References}

[1] Jaiswal J K and Simon S M 2004 Trends Cell Biol. 14497

[2] Huynh W U, Dittmer J J and Alivisatos A P 2002 Science 2952425

[3] Brokmann X, Giacobino E, Dahan M and Hermier J P 2004 Appl. Phys. Lett. 85712

[4] Efros A L and Rosen M 1997 Phys. Rev. Lett. 781110

[5] Gomez D E, van Embden J, Jasieniak J, Smith T A and Mulvaney P 2006 Small 2204

[6] Margolin G, Protasenko V, Kuno M and Barkai E 2006 J. Phys. Chem. B 11019053

[7] Tang J and Marcus R A 2005 J. Chem. Phys. 123054704

[8] Tang J and Marcus R A 2006 J. Chem. Phys. 125044703

[9] Verberk R, Chon J W M, Gu M and Orrit M 2005 Physica E 2619

[10] Verberk R, van Oijen A M and Orrit M 2002 Phys. Rev. B 66233202

[11] Kuno M, Fromm D P, Hamann H F, Gallagher A and Nesbitt D J 2000 J. Chem. Phys. 1123117

[12] Brokmann X, Hermier J P, Messin G, Desbiolles P, Bouchaud J P and Dahan M 2003 Phys. Rev. Lett. 90120601

[13] Biebricher A, Sauer M and Tinnefeld P 2006 J. Phys. Chem. B 1105174

[14] Neuhauser R G, Shimizu K T, Woo W K, Empedocles S A and Bawendi M G 2000 Phys. Rev. Lett. 853301

[15] Empedocles S A, Norris D J and Bawendi M G 1996 Phys. Rev. Lett. 773873

[16] Hohng S and Ha T 2004 J. Am. Chem. Soc. 1261324

[17] Hammer N I, Early K T, Sill K, Odoi M Y, Emrick T and Barnes M D 2006 J. Phys. Chem. B 11014167

[18] Koberling F, Mews A and Basche T 2001 Adv. Mat. 13672

[19] Ray K, Badugu R and Lakowicz J R 2006 J. Am. Chem. Soc. 1288998

[20] Skaff H, Sill K and Emrick T 2004 J. Am. Chem. Soc. 12611322

[21] Odoi M Y, Hammer N I, Sill K, Emrick T and Barnes M D 2006 J. Am. Chem. Soc. 1283506

[22] Frantsuzov P A and Marcus R A 2005 Phys. Rev. B 72155321

[23] Zhang K, Chang H Y, Fu A H, Alivisatos A P and Yang H 2006 Nano Lett. 6843

[24] Schlegel G, Bohnenberger J, Potapova I and Mews A 2002 Phys. Rev. Lett. 88137401

[25] Empedocles S A and Bawendi M G 1999 J. Phys. Chem. B 1031826

[26] Shimizu K T, Woo W K, Fisher B R, Eisler H J and Bawendi M G 2002 Phys. Rev. Lett. 89117401

[27] Pokrant S and Whaley K B 1999 Eur. Phys. J. D 6255

[28] Izrailev F M and Castaneda-Mendoza A 2006 Phys. Lett. A 350355

[29] Chowdhury A, Yu L P, Raheem I, Peteanu L, Liu L A and Yaron D J 2003 J. Phys. Chem. A 1073351 